\title{
Multiscale simulation coupled DRBEM with FVM for the two-phase flow with phase change process of micrometer scale particles
}

\author{
W.-Q. Lu \& K. Xu \\ College of Physical Science, Graduate University of Chinese Academy \\ of Sciences, Beijing, People's Republic of China
}

\begin{abstract}
A multiscale computational method couple dual reciprocity boundary element method (DRBEM) with finite volume method (FVM) is developed, and used to numerically simulate the hydrothermal gasification and evaporation of micrometer scale particles in two-phase flow. The engineering applied background of the problems include: hydrogen production from biomass in supercritical water, water mist fire suppression, etc. Numerical results shows the multiscale computational method coupled DRBEM with FDM is credible and effective. This paper also presents some valuable numerical results for these engineering problems.

Keywords: multiscale computational method, DRBEM, FVM, hydrogen production by biomass, water mist fire suppression.
\end{abstract}

\section{Introduction}

The two-phase flow with evaporation and gasification process of micrometer scale particles occurs in some engineering and scientific problems. The hydrogen production process by biomass particles is implemented in the column reactor with electric heating wall. The uniform mixture of supercritical water and biomass particles continuously flows into the reactor. Hydrogen and other flammable gases are produced by the hydrothermal gasification of biomass particles. There exist complex process of two phase flow, heat transfer and hydrothermal gasification of biomass particles. The process of water mist fire suppression is that micrometer scale water droplets are jetted into high 
temperature environment. The moving water droplets continuously absorb heat from the environment and are vaporized, so that depresses the environment temperature and wins fire suppression.

The main object of this paper is to explore the multiscale numerical method to solving these problems, and open out some valuable results for these engineering practical problems.

\section{Physical models}

Figure 1 is the sketch map of hydrogen production from biomass particles. The reactor is a cylindrical tube with the length $650 \mathrm{~mm}$ and the radius $3 \mathrm{~mm}$. The wall temperature keeps $650^{\circ} \mathrm{C}$. The volume fraction and radius of biomass particles at inlet are 0.03 and $0.15 \sim 0.35 \mathrm{~mm}$, respectively. Hydrothermal gasification of biomass particles occurs in supercritical water. The products of gas flow out at right end of the tube.

Figure 2 is the sketch map of water mist fire suppression. In the domain of the high $3 \mathrm{~m}$ with infinite length and width, spray nozzle is fixed at $2.5 \mathrm{~m}$ high over ground. The micro water droplets and air simultaneously are ejected. The initial radius and velocity of the droplets are $250 \mu \mathrm{m}$ and $1 \mathrm{~m} / \mathrm{s}$, respectively. The mass ratio between air and droplets is 0.5 . The air velocity is $0.01 \mathrm{~m} / \mathrm{s}$ at spray nozzle. The top wall temperature keeps $400 \mathrm{~K}$ and the bottom temperature keeps $350 \mathrm{~K}$.

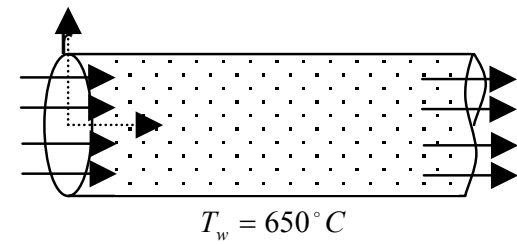

Figure 1: Schematic hydrogen production.

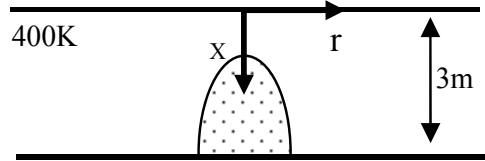

$350 \mathrm{~K}$

Figure 2: Schematic water mist fire suppression.

In both processes, all micro particles are assumed as spherical particles. In numerical method, it needs to consider the numerical models of particles, fluids and their coupling. In order to simplify computation, the two flow fields are considered as axial symmetrical and steady.

\subsection{The gasification model of biomass particles}

Hydrothermal gasification of biomass particles in supercritical water are chemical reaction processes which simultaneously occur at its surface and inside. It is assumed that the gasification of single particle is the process to gradually increase its porosity and decrease its density.

Many researches on the mechanism of the chemical reaction about gasification of biomass particles in supercritical water have been made up to now [1]. In this paper, some simplified assumptions are made: the main 
component of biomass is assumed as cellulose, it can be rapidly transform into glucose in supercritical water, then gasified. In addition, few products of multi carbon gases $\mathrm{C}_{2} \mathrm{H}_{4}$ and $\mathrm{C}_{2} \mathrm{H}_{6}$, etc can be neglected. The mechanism of glucose gasification reaction is summed up on the basis of experimental data [2,3], it can be expressed as the following equation:

$$
\mathrm{C}_{6} \mathrm{H}_{10} \mathrm{O}_{5}+4.5 \mathrm{H}_{2} \mathrm{O} \rightarrow 4.5 \mathrm{CO}_{2}+7.5 \mathrm{H}_{2}+0.5 \mathrm{CO}+\mathrm{CH}_{4}
$$

Consider a micro control volume of particle's inner, its energy balance equation in cylindrical coordinates (see Fig. 3) can be written as the follows:

$$
\frac{\partial}{\partial t}\left(\rho_{s} c_{p, s} T_{s}\right)=k_{s}\left(\frac{\partial^{2} T_{s}}{\partial r^{2}}+\frac{1}{r} \frac{\partial T_{s}}{\partial r}+\frac{\partial^{2} T_{s}}{\partial z^{2}}\right)+(-\Delta H)\left(-\frac{\partial \rho_{s}}{\partial t}\right)
$$

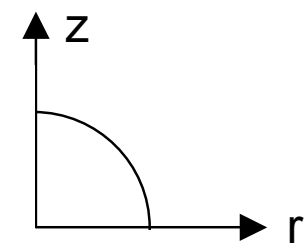

Figure 3: Computational domain of particle's quarter-sphere.

Boundary Condition:

$$
-k_{s}\left(\frac{\partial T_{s}}{\partial r}\right)_{r=R}=h\left(T_{s}-T_{f}\right)
$$

where, $\rho_{s}$ and $T_{s}$ are the local density and temperature of the particle. $\Delta H$ is reaction heat. $h$ is the convective heat transfer coefficient. $T_{f}$ is fluid temperature.

\subsection{The evaporation model of water droplet}

Moving droplet is continually vaporized since convective heat transfer from surrounding fluid. This is a phase change process which occurs at the surface of the droplet. Its energy equation can be written as the follows:

$$
\frac{\rho_{l} c_{p l}}{k_{l}} \frac{\partial T_{l}}{\partial t}=\frac{\partial^{2} T_{l}}{\partial r^{2}}+\frac{1}{r} \frac{\partial T_{l}}{\partial r}+\frac{\partial^{2} T_{l}}{\partial z^{2}}
$$

Boundary condition at the surface:

$$
-k_{l} A \frac{d T_{l}}{d r}+h A\left(T_{f}-T_{l}\right)=-L \dot{m} \quad r=r_{w}
$$


where, $\rho_{l}$ and $T_{l}$ are the density and temperature of the droplet. $L$ is the latent heat of evaporation. $A$ is the surface area of the particle. $h$ is the convective heat transfer coefficient. $\dot{m}$ is the evaporation rate of the droplet: $\dot{m}=\rho_{l} 4 \pi r^{2} d r / d t$. The rate change of the droplet radius is [4]:

$$
\frac{d r}{d t}=-\frac{\rho_{f}}{\rho_{l}} \frac{D_{f}}{2 r} S h \ln (1+B)
$$

where, $D_{f}$ and $S h$ are the coefficient of mass diffusion and Sherwood number, respectively. $B$ is the mass transfer number.

\subsection{The motion equation of a particle}

$$
m_{d} \frac{d \mathbf{v}}{d t}=C_{D} \rho_{f}\left(\mathbf{u}_{f}-\mathbf{v}\right)\left|\mathbf{u}_{f}-\mathbf{v}\right| \frac{A_{d}}{2}+m_{d} \mathbf{g}
$$

where, $m_{d}$ and $A_{d}$ are the mass and surface area of particle, respectively. $\mathbf{u}_{f}$ and $\mathbf{V}$ are the velocity of fluid and particle respectively. The drag coefficient $C_{D}$ is given by the following expression [4]:

$$
C_{D}= \begin{cases}24\left(1+0.166 \mathrm{Re}^{2 / 3}\right) / \mathrm{Re} & \mathrm{Re}<1000 \\ 0.424 & \mathrm{Re}>1000\end{cases}
$$

where $\operatorname{Re}=\rho_{f} d_{p}\left|\mathbf{u}_{f}-\mathbf{v}\right| / \mu_{f}$.

Particle's track can be determined from:

$$
\mathbf{x}=\mathbf{x}_{\mathbf{d}, \mathbf{0}}+\left(\mathbf{v}+\mathbf{v}_{0}\right) \Delta t / 2
$$

where $\mathbf{x}_{\mathbf{d}, \mathbf{0}}$ is the droplet position at the beginning of the time increment.

\subsection{The controlling equations of fluid}

The controlling equations are axial symmetric steady N-S equations:

$$
\frac{\partial}{\partial x}\left(\rho_{f} u \Phi\right)+\frac{1}{r} \frac{\partial}{\partial r}\left(r \rho_{f} \vee \Phi\right)=\frac{\partial}{\partial x}\left(\Gamma_{\Phi} \frac{\partial \Phi}{\partial x}\right)+\frac{1}{r} \frac{\partial}{\partial r}\left(r \Gamma_{\Phi} \frac{\partial \Phi}{\partial r}\right)+S_{\Phi}+S_{\Phi}^{p}
$$

where, when $\Phi$ are given as different values, equations (8) are expressed as mass, momentum, energy, turbulent kinetic energy and the dissipation rate equations, respectively. $S_{\Phi}^{p}$ is the source term produced by particles. Table 1 expresses the corresponding parameter values for different equations.

where, $G_{k}=\mu_{\text {eff }}\left\{2\left[\left(\frac{\partial u}{\partial x}\right)^{2}+\left(\frac{\partial v}{\partial r}\right)^{2}+\left(\frac{v}{r}\right)^{2}\right]+\left(\frac{\partial u}{\partial r}+\frac{\partial v}{\partial x}\right)^{2}\right\}, \mu_{e f f}$ is effective turbulent viscosity coefficient. $\sigma_{k}, \sigma_{\varepsilon}, \sigma_{h}, \sigma_{Y}, c_{1}$ and $c_{2}$ are experience 
constants. $S_{m}, S_{u}, S_{v}, S_{h}$ and $S_{Y}$ are the source terms of mass, momentum, energy and species produced by particles.

In the calculation of the species in hydrogen production process, employing equation (1) to calculate gas production: $\mathrm{CO}, \mathrm{CO}_{2}, \mathrm{H}_{2}, \mathrm{CH}_{4}$. In addition, the water gas shift reaction is also considered:

$$
\mathrm{CO}+\mathrm{H}_{2} \mathrm{O} \rightarrow \mathrm{CO}_{2}+\mathrm{H}_{2}
$$

This is a reversible reaction. However, since the concentration of water is more than that of other species, therefore the above single-direction reaction is only considered.

Table 1: $\quad$ Corresponding parameters for N-S equations.

\begin{tabular}{|c|c|c|c|c|}
\hline $\begin{array}{l}\text { Equa- } \\
\text { tions }\end{array}$ & $\Phi$ & $\Gamma_{\Phi}$ & $S_{\Phi}$ & $S_{\Phi}^{p}$ \\
\hline Mass & 1 & 0 & 0 & $S_{m}$ \\
\hline $\begin{array}{l}\text { Axial } \\
\text { momen- } \\
\text { tum }\end{array}$ & $u$ & $\mu_{e f f}$ & $-\frac{\partial p}{\partial x}+\frac{\partial}{\partial x}\left(\mu_{e f f} \frac{\partial u}{\partial x}\right)+\frac{1}{r} \frac{\partial}{\partial r}\left(r \mu_{e f f} \frac{\partial v}{\partial x}\right)$ & $S_{u}$ \\
\hline $\begin{array}{l}\text { Radial } \\
\text { momen- } \\
\text { tum }\end{array}$ & $v$ & $\mu_{e f f}$ & $-\frac{\partial p}{\partial r}+\frac{\partial}{\partial x}\left(\mu_{e f f} \frac{\partial u}{\partial r}\right)+\frac{1}{r} \frac{\partial}{\partial r}\left(r \mu_{e f f} \frac{\partial v}{\partial r}\right)-2 \mu_{e f f} \frac{v}{r^{2}}$ & $S_{v}$ \\
\hline $\begin{array}{l}\text { Kinetic } \\
\text { energy }\end{array}$ & $k$ & $\mu_{e f f} / \sigma_{k}$ & $G_{k}-\rho \varepsilon$ & 0 \\
\hline $\begin{array}{l}\text { Dissipa- } \\
\text { tion rate }\end{array}$ & $\varepsilon$ & $\mu_{e f f} / \sigma_{\varepsilon}$ & $\frac{\varepsilon}{k}\left(c_{1} G_{k}-c_{2} \rho \varepsilon\right)$ & 0 \\
\hline $\begin{array}{l}\text { Enthal- } \\
\text { py }\end{array}$ & $h$ & $\mu_{e f f} / \sigma_{h}$ & 0 & $S_{h}$ \\
\hline Species & Y & $\mu_{e f f} / \sigma_{Y}$ & 0 & $S_{Y}$ \\
\hline
\end{tabular}

\section{Numerical methods}

Since the volume fractions of particles are small in these problems, particles are dispersed in fluids. Employing the two fluids model of two-phase flow to calculate these problems is not appropriate. The model of dispersive particles groups is considered in this calculation. In this model, it needs to calculate the behaviors of the dispersive particles groups and fluid. A multiscale computational method coupled macroscopic fluid fields with micro particle fields is constructed as the follows.

\subsection{The calculation of micro particle fields}

Our improved axisymmetric DRBEM (ADRBEM) [5] is used to solve equation (2) and (3) for both different problems respectively. The choice of 
function $f$ is very important for the calculation by DRBEM. However, in early ADRBEM, it is impossible to arrange nodes on the symmetrical axis since the singularity of function $f$ early used in ADRBEM. In reference [5], the singularity is avoided by integral averaging and selecting different assemblage of functions $f$. In this calculation, the marked B-02 and B-05 type $f$ function [5] are used to calculate biomass particle and droplet, respectively.

B-02:

$$
\begin{gathered}
r \neq 0: f=1-\frac{1}{3} \frac{r_{i}}{r}+\hat{p}\left(1-\frac{1}{4} \frac{r_{i}}{r}\right)+\hat{p}^{2}\left(1-\frac{1}{5} \frac{r_{i}}{r}\right), \hat{p}=\sqrt{\left(r-r_{i}\right)^{2}+\left(z-z_{i}\right)^{2}} \\
r=0: f=1+\hat{p}\left(1-\frac{1}{4} \frac{r_{i}^{2}}{\hat{p}^{2}}\right)+\hat{p}^{2}\left(1-\frac{2}{5} \frac{r_{i}^{2}}{\hat{p}^{2}}\right), \hat{p}=\sqrt{r_{i}^{2}+\left(z-z_{i}\right)^{2}}
\end{gathered}
$$

B-05:

$r \neq 0$ :

$$
\begin{aligned}
& f=1-\frac{1}{3} \frac{r_{i}}{r}+\hat{p}\left(1-\frac{1}{4} \frac{r_{i}}{r}\right)+\hat{p}^{2}\left(1-\frac{1}{5} \frac{r_{i}}{r}\right)+\hat{p}^{3}\left(1-\frac{1}{6} \frac{r_{i}}{r}\right)+\hat{p}^{4}\left(1-\frac{1}{7} \frac{r_{i}}{r}\right)+\hat{p}^{5}\left(1-\frac{1}{8} \frac{r_{i}}{r}\right), \\
& \hat{p}=\sqrt{\left(r-r_{i}\right)^{2}+\left(z-z_{i}\right)^{2}} \\
& r=0: \\
& \quad f=1+\hat{p}\left(1-\frac{1}{4} \frac{r_{i}^{2}}{\hat{p}^{2}}\right)+\hat{p}^{2}\left(1-\frac{2}{5} \frac{r_{i}^{2}}{\hat{p}^{2}}\right)+\hat{p}^{3}\left(1-\frac{1}{2} \frac{r_{i}^{2}}{\hat{p}^{2}}\right)+\hat{p}^{4}\left(1-\frac{4}{7} \frac{r_{i}^{2}}{\hat{p}^{2}}\right)+\hat{p}^{5}\left(1-\frac{5}{8} \frac{r_{i}^{2}}{\hat{p}^{2}}\right) \\
& \quad \hat{p}=\sqrt{r_{i}^{2}+\left(z-z_{i}\right)^{2}}
\end{aligned}
$$

The inner temperature distributions of two different kinds of particles are obtained respectively. The variations of biomass particle's density and droplet size are further gotten respectively. It is noticed that the differences between the calculations in two different kinds of particles exist as the follows.

In equation (2), $\partial \rho_{s} / \partial t=-k_{g} \rho_{s}$, where, $k_{g}$ is the coefficient of the reaction $[2,3]$ :

$$
k_{g}=-10^{3.09 \pm 0.26} \exp (-63.6 \pm 3.9 / R T)
$$

Since equation (2) is nonlinear, Newton iterative method is used to DRBEM calculation.

In equation (3), the mass transfer number $B$ is the function of particle's temperature, therefore $\dot{m}$ is also correlated with particle's temperature. Hence boundary condition ( $3 \mathrm{~b})$ is nonlinear. In order to simplify the calculation, boundary condition ( $3 \mathrm{~b})$ is transformed into:

$$
-k_{l} \frac{d T_{l}}{d r}=h^{\prime}\left(T_{l}-T_{f}\right) \quad r=r_{w}
$$

where, $h^{\prime}$ is effective coefficient of convective heat transfer, which can be calculated by iteration. 
After solving equations (5-7), the velocities and tracks of particles are gotten. Finally, the source terms $S_{\Phi}^{p}$ of particle are calculated by particle- source-in cell (PSIC) method [6].

\subsection{The calculation of macroscopic fluid fields}

Finite volume method with SIMPLEC scheme is used to solving equations (8). All physical quantities of macroscopic fluid fields are obtained.

\subsection{The couple calculation of both fields}

Macroscopic fluid fields and micro particle fields are coupled by the source terms $S_{\Phi}^{p}$. This couple computation is an iterative calculation between fluid fields and the source terms of particles.

\section{Numerical results}

\subsection{Hydrogen production by biomass particles in supercritical water}

Figure 4 shows the comparison between experimental data [7] and numerical results of main gas production under the conditions: particles radius $0.2 \mathrm{~mm}$, reaction time $0.5 \mathrm{~min}$. As shown in Fig. 4 , the better agreement between both results indicates the models and methods are credible.

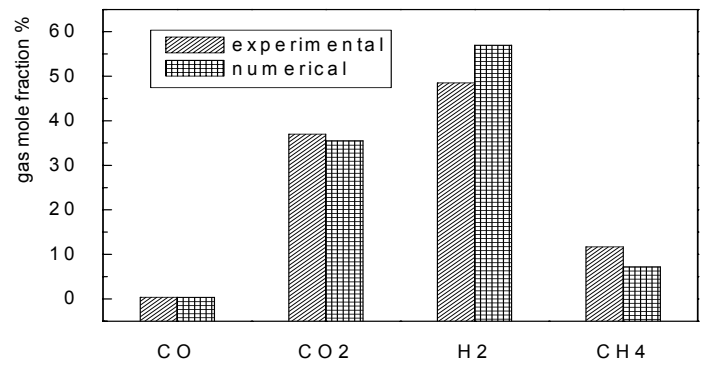

Figure 4: The numerical gas mole fraction compared with experimental data.

Figure 5 shows the effect of the wall temperature on the mole fraction of gas production in particles radius $0.2 \mathrm{~mm}$. As shown in the figure, $\mathrm{H}_{2}$ increases with increasing temperature, but $\mathrm{CO}$ is decreased with increasing temperature. The reason is when temperature is higher, the rate of water gas shift reaction (9) between water and vapor is rapider. In the case of enough water, $\mathrm{CO}$ is consumed very much, and simultaneously $\mathrm{H}_{2}$ is produced.

Figure 6 depicts the variation of hydrogen yield with particle radius under same flux, temperature and mass fraction. As shown in the figure, Hydrogen yield is decreased with increasing particle radius. This is since under same flux and mass fraction, the smaller the particle is, the more the particle's number is, 
so that the interactional area between water and particles increase. This induces the hydrogen yield increases.

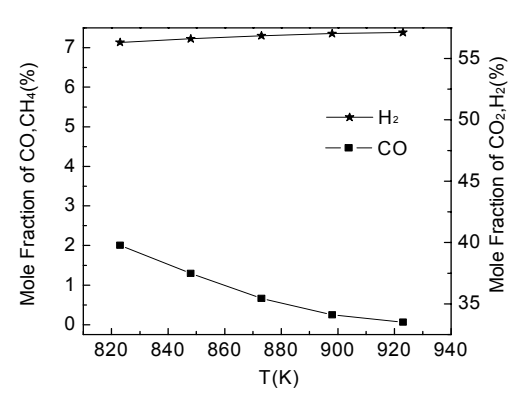

Figure 5: $\quad$ Effect of reactor temperature on mole fraction of gas.

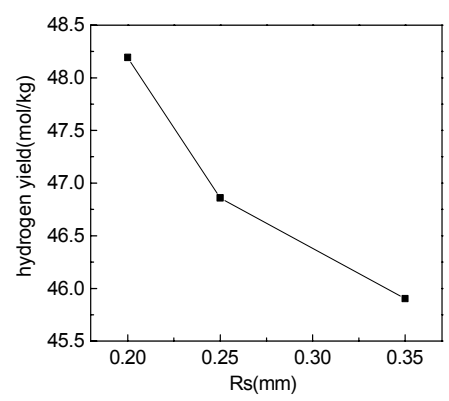

Figure 6: Effect of particle size on hydrogen yield.

\subsection{Water spraying}

Figure 7 shows the velocity field of the fluid. In neighborhood of the nozzle, the motion of fluid is dragged by downward moving particles, which induces to form an anticlockwise vortex. In the radial location $20-30 \mathrm{~m}$, the velocity field keeps invariable. Therefore the radial location over $20 \mathrm{~m}$ can be considered as infinite beyond.

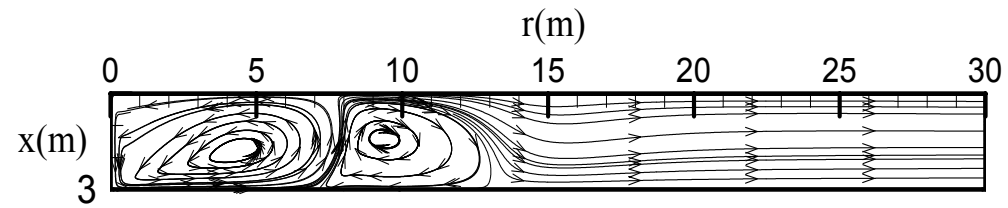

Figure 7: Velocity vector map of fluid.

Figure 8 depicts the temperature field of fluid. The low temperature domain in neighborhood of central axis is produced since here the concentration of droplet is higher, and the plentiful quantity of heat is absorbed by evaporation, that decreases local temperature.

Figure 9 shows a moving track of a particle and the flow field in neighborhood of central axis. As shown in the figure, initial moving track of the particle is near parabola, afterward its track gradually tends to local streamline of fluid. This reason is that the radius of the particle becomes gradually small since evaporation, and the effect of fluid motion on the particle is gradually enhanced.

Figure 10 depicts the variation of water drip's radius with time. As shown in the figure, the variation velocity of the drip size is slower and slower. This 
reason is that the speed of evaporation becomes slower and slower with the drop moving. This is also corresponded to the temperature variation of environment as shown in Fig. 8.

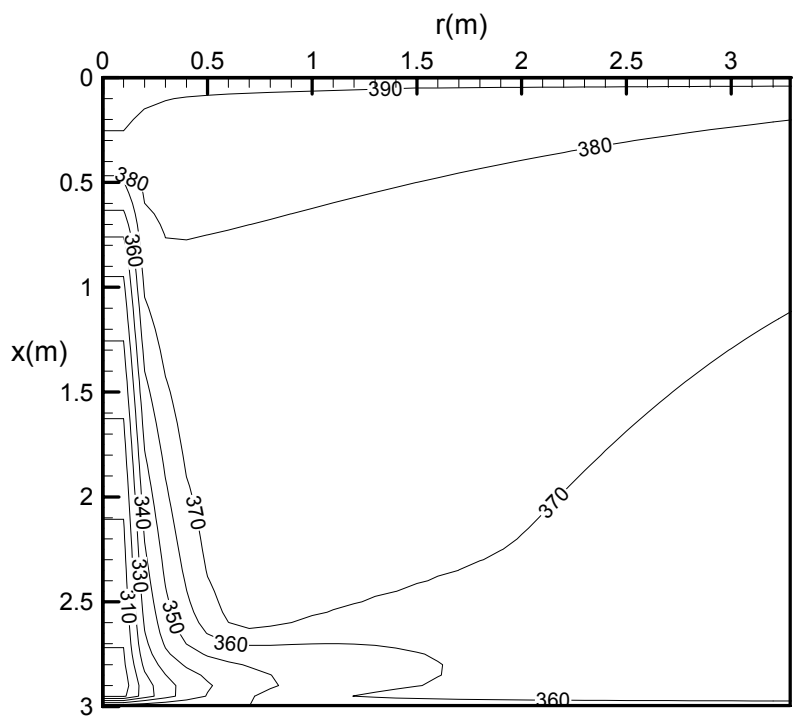

Figure 8: The temperature field of fluid.
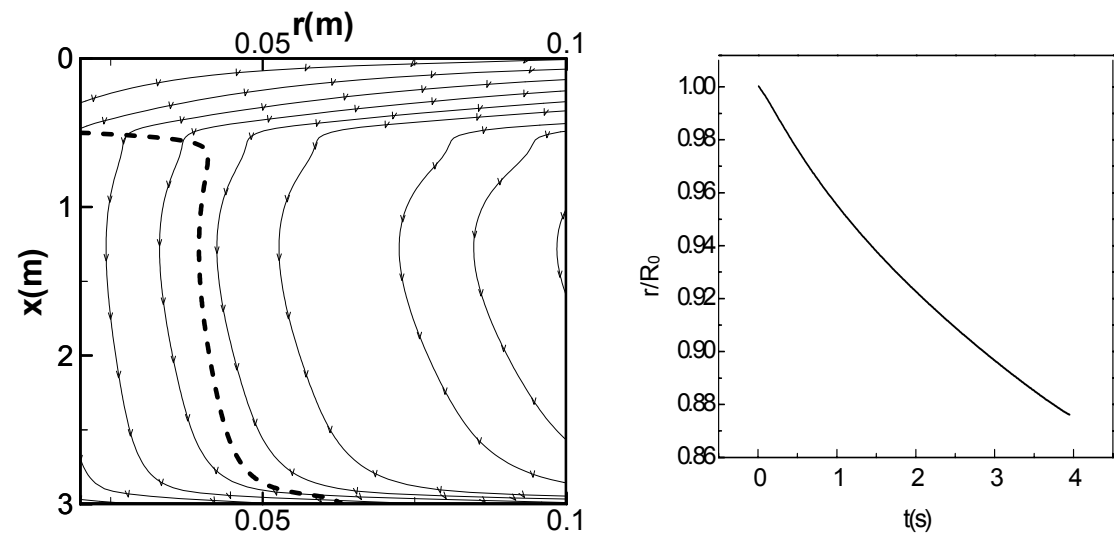

Figure 9: Moving track of particle and Figure 10: The variation of water flow field (particle's track). droplet radius. 


\section{Conclusions}

The multiscale computational method coupled macroscopic fluid fields by FVM with micro particles fields by DRBEM is developed and used to numerically simulate the hydrogen production by biomass particles in supercritical water and water spraying. The hydrothermal gasification and evaporation of dispersivephase particles are successfully simulated respectively. The simulation gives the comparable with experimental data and physical reasonable results of micro particles fields and fluid fields. Present numerical examples express that the method is effective and credible. Some valuable results for these engineering problems are also given.

\section{Acknowledgement}

The project is supported by the National Natural Scientific Foundation of China (Grant 50676102 and 50536030)

\section{References}

[1] Lu, Y.J. \& Guo, L.J., etc, Hydrogen production by biomass gasification in supercritical water: A parametric study. International Journal of Hydrogen Energy 31, pp. 822-831, 2006.

[2] Wei, F. \& Hedzer, J., etc, Biomass conversions in subcritical and supercritical water: driving force, phase equilibrium, and thermodynamic analysis. Chemical Engineering and Processing, 43, pp. 1459-1467, 2004.

[3] Lee, I. \& Kim, M.., etc, Gasification of glucose in supercritical water. Ind. Chem. Eng. Res. 41, pp. 1182-1188, 2002.

[4] Bracco, F.V. Modelling of engine sprays, SAE Paper 850394, 1985.

[5] Bai, F.W. \& Lu,W.-Q., The selection and assemblage of approximation functions and disposal of its singularity in axisymmetric DRBEM for heat transfer problems. Engineering Analysis with Boundary Elements, 28, pp. 955-965, 2004.

[6] Crowe, C.T. \& Sharma, M.P., etc, The Particle-Source-In Cell(PSI-CELL) Model for Gas-Droplet Flows. Journal of Fluids Engineering, 99, pp. 325330, 1977.

[7] Lv, Y.J. \& Guo, L.J., etc, Experimental research of hydrogen production from biomass particles gasification in supercritical water, The National Great Basic Research Development Project of China (Grant 2003CB214500) Research Paper 3-06, 2004. 